\title{
Spatial Free Vibration Analysis for Truck-Mounted Howitzer
}

\author{
Ragaee Ahmed Rateb, Yang Guolai, and Ge Jianle
}

\begin{abstract}
The main issue addressed in this article is the lays out of truck mounted howitzer free vibration theory as a continuous interconnected multi-body rigid model in spatial coordinates. The mathematical modeling is developed by the method of the first kind of Lagrange's equations based on the weapon system discretization techniques. Searching for the eigen vibration characteristics, the characteristic equation is solved based on the derived mathematical model. This simulation numerical result is validated by certain comparative experimental works. Finally, the sensitivity analysis by using random optimization theory is applied on the simulation model in order to find the weapon system optimal design layout. This study shows a new spatial investigation applied on truck mounted howitzer. In addition, the sensitivity analysis compels the designer to identify the critical variables affect the vibration behavior of this artilary weapon system.
\end{abstract}

Index Terms-Boundary conditions, military trucks, natural frequencies, sensitivity analysis.

\section{INTRODUCTION}

Particularly in truck dynamics, the multibody techniques can be used as an efficient tool for the design and analysis process [1], [2]. The constructed mechanical model is represented as a system of rigid or elastic bodies and massless interconnections [3]. There are some differences between military and civilian trucks in terms of shape, type and application, but of course, they match in some parameters [4]. Military trucks (MT) vary greatly in size, power, and configurations [5] as shown in Fig. 1. Actually, the MT share many common constructions such as driver cabinet, engine, chassis, suspensions, tires and axes besides many associated systems as hydraulic, pneumatic, and electrical systems as presented in the same figure.

The vibration behavior of the MT, which is called the MT vibration ride comfort, is highly dependent on the natural frequencies and mode shapes which can be constructed through multibody modeling concepts [6]. In self-propelled artillery weapon system, the cannons are loaded on tracked vehicles while usually MT are used to trail cannons in the battlefield. Now the MT are used to load the artilleries as an effect of great developments in their capacities and capabilities, in addition to the strong traction motors generations used by this type of MT. The advantages of using

Manuscript received in January 10, 2015; revised April 1, 2016. This work was supported in part by the chines Department of Commerce under Grant BS123456.

Ragaee Ahmed Rateb is with School of Mechanical Engineering, Nanjing University of Science and Technology, Nanjing, China (e-mail: ragaee2010@yahoo.com).

Yang GuoLai is with Nanjing University of Science and Technology, China (e-mail: yyanggl@mail.njust.edu.cn).
MT to transport cannons can be summed up in the high-speed mobility, rapid deployment, and AWS cost reduction requirements. The MT used to transport cannons have many added parts such as tilting legs, gun housing, gun frame, ammunition housing, bombardment, mine detonation system...etc. By virtue of the MT works in the battlefield, it must be more rigid and more reliable comparing with the civilian one. Thus, the assumption of the continuous rigid multibody model is acceptable perception. Also, the weight must be at the slightest degree to grantee the mobility and maneuverability.

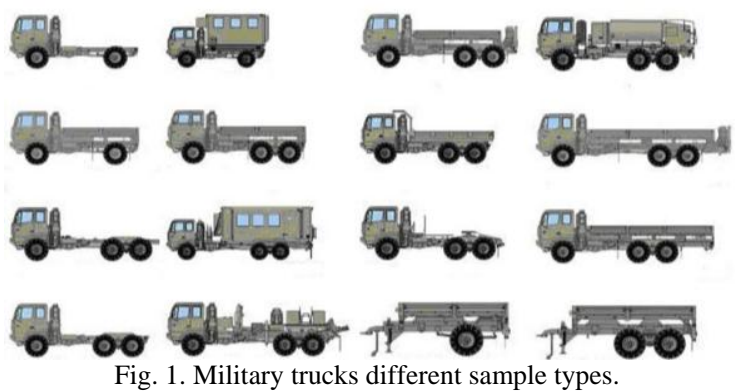

There are different studies in civilian truck dynamics investigations especially in planner representation while in the case of the MT, there are very view available studies especially truck mounted howitzer due to their application limitations and modernists [7]. One of the most important studies in truck dynamics is the work done by B. Simeon and other [8]; they presented a planar vertical truck model with nonlinear suspension and its multibody system formulation. They deal with the selected truck as completely rigid and continuous multibody model which provide a complete investigation for the represented test problem. A simple planner presentation including the truck chassis flexibility nature is the main insufficient survey in this work. I. M. Ibrahim and other [9] studied the vibration behavior of trucks, in particular, the effect of including a flexible frame. They studied the effects of the frame flexibility by modeling the truck frame using the finite element method. By comparing the responses of the rigid and flexible body models, they proof that the frame flexibility strongly affects the accelerations of both driver and the truck body. Another important study provided by Ragaee A. Rateb and other [10] deal with a completely flexible truck-mounted howitzer in which the flexible model was generated by the fundamentals of finite element techniques through using Hypermesh software. They calculate the modal analysis for such weapon system to validate the constructed finite element model and apply the sensitivity analysis by random optimization theory searching for the optimal weapon system layout. A more detailed study was done by Cai WenYong in his Ph.D. thesis 
[11], [12]. He studied the launching dynamics problem of truck-mounted howitzer in firing by the theory of flexible multi-body dynamics. He constructed the dynamics model of flexible bodies system in which both the barrel and the upper carriage of the cannon system are treated as the flexible bodies. He considered the carrier truck and the other weapon system components as a continuous rigid unit. The sensitivity analysis model for the flexible multibody system dynamics was built by the random variation method. On the other hand, Yu Hailong and Xiaoting Rui [13] generate a new theory for studying the multi-body system dynamics represents artillery weapon system. They developed field transfer matrix of an arbitrary rigid body vibrating in the linear range and the transfer matrix method of multi-body system dynamics. They study the natural vibration of a general multi-body gun system. They also construct the transfer equation and transfer matrix of a corresponding multi-body gun system, then they developed the analytical form of frequency equation and the modal functions for such system.

This article represents the complete weapon system dynamic investigation in the spatial presentation by the method of Lagrange's equations. This will provide the theoretic basis for the system design, dynamic analysis, and finalizing the layout of the vehicle mounted howitzer. Also, the applied sensitivity analysis for the constructed multibody model will help to increase the design robustness and to identify the critical parameters that affect the truck vibration.

\section{WEAPON SYSTEM COMPOSITION AND PROBLEM FORMULATION}

\section{A. Truck Mounted Howitzer Composition}

The weapon system case of study was designed to load 122 mm howitzer cannon on a compatible $6 \times 6$ heavy-duty MT as shown in 3D geometrical model portrayed in Fig. 2. This 3D model was created by "Creo /Parametric 2.0" to represent the required details to formulate the problem outlines. In order to prevent mathematical complexity from obscuring the theoretical concepts, such geometrical model was simplified, especially in both cannon and ammunitions reloading systems, in which their deep details were ignored.

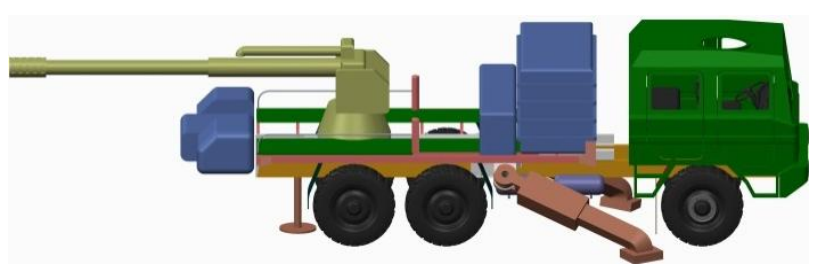

Fig. 2. 3D model for the truck mounted howitzer case of study.

Such weapon system as shown in Fig. 2 consists of; 122 $\mathrm{mm}$ howitzer cannon system including aiming and firing mechanisms, gun equilibrator, front and rear ammunition reloads systems and charge depots, hydraulic deployment system consists of 3 fixation legs in which 2 of them in the front layout position and another one in the back position, beside carrier $6 \times 6$ special purpose MT including driver cabinet and control room, traction motor with front and back engine blocks, truck lower and upper chassis, truck main body and main corridors, tiers and tires axes, truck suspension system, fuel tank, and oil tank.

\section{B. Problem Formulation}

However, due to the military specifications, the MT chassis must be rigid enough to prevent any unexpected vibration in both maneuvering and launching phases. Therefore, and without error in the assumption objectivity, the carrier truck can be assumed as continuous rigid manner. The discretized system with its abbreviations includes; 6 tiers mass blocks " $t$ ", 6 suspension springs " $S$ ", driver room including engine blocks " $d v$ ", truck main body “ $T$ ”, gun system " $g$ ”, front ammunition depots " $f p$ ”, rear ammunition depots " $r p$ ", filled oil tank block " $o$ ", filled fuel tank block " $f$ ", sides and back fixation feet mass blocks " $f e$ ", linear massless springs, and required occupying dampers represent both massless interconnections between different model components and carrier main body. Fig. 3. shows a complete schematic vibrating model for such weapon system by using the previous abbreviations and assumptions including the selected origin point and the reference coordinates. This model includes the bounce $x$, the roll $\theta$, the pitch $\varphi$ for each discretized component beside the wheels hop and the other masses blocks hop, like the fuel tank.

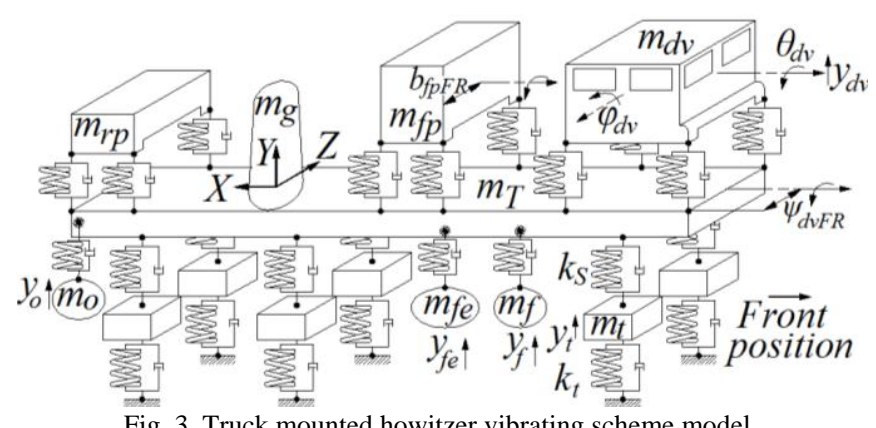

Fig. 3. Truck mounted howitzer vibrating scheme model.

Table I depicts the definitions for the selected generalized coordinates and the design abbreviation definitions.

TABLE I: THE SELECTED ABBREVIATION DEFINITIONS

\begin{tabular}{ll}
\hline \hline Abbreviation & Definition \\
\hline$\{X, Y, Z\},\{x, y, z\}$ & Global and Local Cartesian coordinates respectively \\
$B, M, F$ & Back, Middle, and Front position respectively \\
$R, L$ & Right and left position respectively \\
$a, b$ & Longitudinal and Lateral distances between components \\
& center of gravity and its fulcrum points respectively \\
& Longitudinal and Lateral distances between the main \\
& truck body i.e. "Tg" center of gravity and the assembled \\
& components fulcrum points respectively
\end{tabular}

Actually, the connection between various components in the simulation model is a part of welding link or nails, thus, the stiffness simulation value for these springs must be in the maximum available level. As assumptions, the cannon system and the main truck farm will vibrate as one unit continuous manner with abbreviation " $T g$ ", and the deviations in all mass blocks and main components linear paths "which supposed to be shaking in a linear nature such 
as the fuel tank as a result of main components rotation around their axes" are negligible.

Noting that, rolling and pitching angle variations will be around the discretized block center of gravity longitudinal and lateral axes respectively, in addition to, the sign of vectors represents distance from the point of rotation, i.e. component center of gravity, to the path of position variation must be taken into consideration in the numerical calculations. To prevent the definitions confusion and for further clarification, for example, $K_{S F R}$ represents the Suspension stiffness in the Front Right position, $b_{f p F R}$ represents the lateral distance between the front ammunition depots rotation axis and the Front Right fulcrum point, and $\psi_{d v F R}$ represents the lateral distance between the main vibrated truck body " $T g$ " rotation axis and the driver room Front Right fulcrum point.

\section{Mathematical Modeling and Deriving the Minimum Resonance Frequency}

Based on the previous problem formulation, 23 Degrees of Freedom (DOF) have been defined for 15 components, in addition to 6 fixed holonomic constraints [14] for each tire. The applied Lagrange's equations of the first kind structure are presented in Table. II. In regards of kinetic and potential energies equations, their construction related to such model are calculated in Eq. $(1,2)$ respectively.

\section{TABLE II: STRUCTURE OF LAGRANGE EQUATIONS}

\begin{tabular}{ll}
\hline \hline Definition & Abbreviation and equation \\
\hline $\begin{array}{l}\text { Generalized coordinates, velocities, and } \\
\text { admissible variations respectively }\end{array}$ & $q_{i}, \dot{q}_{i}, \delta q_{i}$ \\
Generalized non conservative force & $Q_{i}$ \\
Virtual work done & $\delta W=\sum_{i=1}^{n} Q_{i} \cdot \delta q_{i}$ \\
$\begin{array}{l}\text { Total kinetic, and potential energy of the } \\
\text { system respectively }\end{array}$ & $T\left(q_{i}, \dot{q}_{i}\right), V\left(q_{i}\right)$ \\
$\begin{array}{l}\text { Lagrangian } \\
\text { Lagrange's Equations to construct } \\
\text { equation of motion }\end{array}$ & $\left.\frac{d}{d t}\left\lfloor\frac{\partial L}{\partial \dot{q}_{i}}\right\rfloor-\frac{\partial L}{\partial q_{i}}=\dot{q}_{i}\right)=Q_{i}$ \\
\hline \hline
\end{tabular}

$$
\begin{aligned}
T= & \frac{1}{2} m_{t}\left[\dot{y}_{t B R}^{2}+\dot{y}_{t B L}^{2}+\dot{y}_{t M R}^{2}+\dot{y}_{t M L}^{2}+\dot{y}_{t F R}^{2}+\dot{y}_{t F L}^{2}\right] \\
& +\frac{1}{2} m_{f}\left[\dot{y}_{f}+\dot{y}_{T g}-\psi_{f} \dot{\theta}_{T g}+\zeta_{f} \dot{\varphi}_{T g}\right]^{2} \\
& +\frac{1}{2} m_{o}\left[\dot{y}_{o}+\dot{y}_{T g}-\psi_{o} \dot{\theta}_{T g}-\zeta_{o} \dot{\varphi}_{T g}\right]^{2} \\
& +\frac{1}{2} m_{f e R}\left[\dot{y}_{f e R}+\dot{y}_{T g}-\psi_{f e R} \dot{\theta}_{T g}+\zeta_{f e R} \dot{\varphi}_{T g}\right]^{2} \\
& +\frac{1}{2} m_{f e L}\left[\dot{y}_{f e L}+\dot{y}_{T g}+\psi_{f e L} \dot{\theta}_{T g}+\zeta_{f e L} \dot{\varphi}_{T g}\right]^{2} \\
& +\frac{1}{2} m_{f e B}\left[\dot{y}_{f e B}+\dot{y}_{T g}+\psi_{f e B} \dot{\theta}_{T g}-\zeta_{f e B} \dot{\varphi}_{T g}\right]^{2} \\
& +\frac{1}{2}\left[m_{T g} \dot{y}_{T g}^{2}+I_{X T g} \dot{\theta}_{T g}^{2}+I_{Z T g} \dot{\varphi}_{T g}^{2}\right] \\
& +\frac{1}{2} m_{d v}\left[\dot{y}_{d v}+\dot{y}_{T g}+\zeta_{d v} \dot{\varphi}_{T g}\right]^{2} \\
& +\frac{1}{2} m_{f p}\left[\dot{y}_{f p}+\dot{y}_{T g}+\zeta_{f p} \dot{\varphi}_{T g}\right]^{2} \\
& +\frac{1}{2} m_{r p}\left[\dot{y}_{r p}+\dot{y}_{T g}-\zeta_{r p} \dot{\varphi}_{T g}\right]^{2} \ldots
\end{aligned}
$$

$$
\begin{aligned}
& +\frac{1}{2}\left[I_{x d v} \dot{\theta}_{d v}^{2}+I_{z d v} \dot{\varphi}_{d v}^{2}\right]+\frac{1}{2}\left[I_{x f p} \dot{\theta}_{f p}^{2}+I_{z f p} \dot{\varphi}_{f p}^{2}+\right] \\
& +\frac{1}{2}\left[I_{x r p} \dot{\theta}_{r p}^{2}+I_{z r p} \dot{\varphi}_{r p}^{2}\right] \\
& +\frac{1}{2} \dot{\theta}_{T g}^{2}\left[I_{X f}+I_{X o}+I_{X f e R}+I_{X f e L}+I_{X f e B}\right] \\
& +\frac{1}{2} \dot{\varphi}_{T g}^{2}\left[I_{Z f}+I_{Z o}+I_{Z f e R}+I_{Z f e L}+I_{Z f e B}\right] \\
& +\frac{1}{2} \dot{\theta}_{T g}^{2}\left[I_{X d v}+I_{X f p}+I_{X r p}\right] \\
& +\frac{1}{2} \dot{\varphi}_{T g}^{2}\left[I_{Z d v}+I_{Z f p}+I_{Z r p}\right] \\
& V=\frac{1}{2} K_{t}\left[y_{t B R}^{2}+y_{t B L}^{2}+y_{t M R}^{2}+y_{t M L}^{2}+y_{t F R}^{2}+y_{t F L}^{2}\right] \\
& +\frac{1}{2} K_{S F R}\left[y_{T g}-y_{t F R}-\psi_{t F R} \theta_{T g}+\zeta_{t F R} \varphi_{T g}\right]^{2} \\
& +\frac{1}{2} K_{S F L}\left[y_{T g}-y_{t F L}+\psi_{t F L} \theta_{T g}+\zeta_{t F L} \varphi_{T g}\right]^{2} \\
& +\frac{1}{2} K_{S M R}\left[y_{T g}-y_{t M R}-\psi_{t M R} \theta_{T g}+\zeta_{t M R} \varphi_{T g}\right]^{2} \\
& +\frac{1}{2} K_{S M L}\left[y_{T g}-y_{t M L}+\psi_{t M L} \theta_{T g}+\zeta_{t M L} \varphi_{T g}\right]^{2} \\
& +\frac{1}{2} K_{S B R}\left[y_{T g}-y_{t B R}-\psi_{t B R} \theta_{T g}-\zeta_{t B R} \varphi_{T g}\right]^{2} \\
& +\frac{1}{2} K_{S B L}\left[y_{T g}-y_{t B L}+\psi_{t B L} \theta_{T g}-\zeta_{t B L} \varphi_{T g}\right]^{2} \\
& +\frac{1}{2} K_{d v F R}\left[\begin{array}{l}
y_{d v}-b_{d v F R} \theta_{d v}+a_{d v F R} \varphi_{d v} \\
-y_{T g}+\psi_{d v F R} \theta_{T g}-\zeta_{d v F R} \varphi_{T g}
\end{array}\right]^{2} \\
& +\frac{1}{2} K_{d v F L}\left[\begin{array}{l}
y_{d v}+b_{d v F L} \theta_{d v}+a_{d v F L} \varphi_{d v} \\
-y_{T g}-\psi_{d v F L} \theta_{T g}-\zeta_{d v F L} \varphi_{T g}
\end{array}\right]^{2} \\
& +\frac{1}{2} K_{d v B R}\left[\begin{array}{l}
y_{d v}-b_{d v B R} \theta_{d v}-a_{d v B R} \varphi_{d v} \\
-y_{T g}+\psi_{d v B R} \theta_{T g}-\zeta_{d v B R} \varphi_{T g}
\end{array}\right]^{2} \\
& +\frac{1}{2} K_{d v B L}\left[\begin{array}{l}
y_{d v}+b_{d v B L} \theta_{d v}-a_{d v B L} \varphi_{d v} \\
-y_{T g}-\psi_{d v B L} \theta_{T g}-\zeta_{d v B L} \varphi_{T g}
\end{array}\right]^{2} \\
& +\frac{1}{2} K_{f p F R}\left[\begin{array}{l}
y_{f p}-b_{f p F R} \theta_{f p}+a_{f p F R} \varphi_{f p} \\
-y_{T g}+\psi_{f p F R} \theta_{T g}-\zeta_{f p F R} \varphi_{T g}
\end{array}\right]^{2} \\
& +\frac{1}{2} K_{f p F L}\left[\begin{array}{l}
y_{f p}+b_{f p F L} \theta_{f p}+a_{f p F L} \varphi_{f p} \\
-y_{T g}-\psi_{f p F L} \theta_{T g}-\zeta_{f p F L} \varphi_{T g}
\end{array}\right]^{2} \\
& +\frac{1}{2} K_{f p B R}\left[\begin{array}{l}
y_{f p}-b_{f p B R} \theta_{f p}-a_{f p B R} \varphi_{f p} \\
-y_{T g}+\psi_{f p B R} \theta_{T g}-\zeta_{f p B R} \varphi_{T g}
\end{array}\right]^{2} \\
& +\frac{1}{2} K_{f p B L}\left[\begin{array}{l}
y_{f p}+b_{f p B L} \theta_{f p}-a_{f p B L} \varphi_{f p} \\
-y_{T g}-\psi_{f p B L} \theta_{T g}-\zeta_{f p B L} \varphi_{T g}
\end{array}\right]^{2} \\
& +\frac{1}{2} K_{r p F R}\left[\begin{array}{l}
y_{r p}-b_{r p F R} \theta_{r p}+a_{r p F R} \varphi_{r p} \\
-y_{T g}+\psi_{r p F R} \theta_{T g}+\zeta_{f p F R} \varphi_{T g}
\end{array}\right]^{2} \\
& +\frac{1}{2} K_{r p F L}\left[\begin{array}{l}
y_{r p}+b_{r p F L} \theta_{f p}+a_{r p F L} \varphi_{f p} \\
-y_{T g}-\psi_{r p F L} \theta_{T g}+\zeta_{r p F L} \varphi_{T g}
\end{array}\right]^{2} \\
& +\frac{1}{2} K_{r p B R}\left[\begin{array}{l}
y_{r p}-b_{r p B R} \theta_{r p}-a_{r p B R} \varphi_{r p} \\
-y_{T g}+\psi_{r p B R} \theta_{T g}+\zeta_{r p B R} \varphi_{T g}
\end{array}\right]^{2} \quad \cdots
\end{aligned}
$$




$$
\begin{aligned}
& +\frac{1}{2} K_{r p B L}\left[\begin{array}{l}
y_{f p}+b_{f p B L} \theta_{f p}-a_{f p B L} \varphi_{f p} \\
-y_{T g}-\psi_{f p B L} \theta_{T g}+\zeta_{f p B L} \varphi_{T g}
\end{array}\right]^{2} \\
& +\frac{1}{2} K_{f e R}\left[y_{T g}-y_{f e R}-\psi_{f e R} \theta_{T g}+\zeta_{f e R} \varphi_{T g}\right]^{2} \\
& +\frac{1}{2} K_{f e L}\left[y_{T g}-y_{f e L}+\psi_{f e L} \theta_{T g}+\zeta_{f e L} \varphi_{T g}\right]^{2} \\
& +\frac{1}{2} K_{f e B}\left[y_{T g}-y_{f e B}-\psi_{f e L} \theta_{T g}-\zeta_{f e B} \varphi_{T g}\right]^{2} \\
& +\frac{1}{2} K_{f}\left[y_{T g}-y_{f}-\psi_{f} \theta_{T g}+\zeta_{f} \varphi_{T g}\right]^{2} \\
& +\frac{1}{2} K_{o}\left[y_{T g}-y_{o}-\psi_{o} \theta_{T g}-\zeta_{o} \varphi_{T g}\right]^{2}
\end{aligned}
$$

As a result of MT designed nature and masses distributions, the suspension system in the rear and middle axes is stronger than the front axis. Also, to reduce the complexity in the previous constructing equations, assume that any pair of suspensions has the same simulation stiffness values "i.e. $K_{S F R}=K_{S F L}$ ". Of course, this simplification will reduce the complexity of the constructed equation. Theoretically, to calculate the modal analysis, there is no requirement necessity to construct the dissipation function; we just care about the free un-damped oscillations. Anyway, it's easy to construct the dissipation matrix since all occupying dampers are constructed in the simulation model in parallel connection with their buddy springs; simply by Rayleigh dissipation function and similar to Eq. (2) but replace any $K$ by $C$ and any DOF to its derivative. However, based on first kind Lagrange equations and for small linear vibrations, the extend form of Lagrange equation can be rewritten in a simpler and more practical applicable Lagrange as Eq. (3).

$$
\frac{d}{d t}\left\lfloor\frac{\partial T}{\partial \dot{q}_{i}}\right\rfloor-\frac{\partial T}{\partial q_{i}}+\frac{\partial D}{\partial \dot{q}_{i}}+\frac{\partial V}{\partial q_{i}}=Q_{i}
$$

The previous equation can be used to provide the equations of motion in a matrix form which represents the constructed model as shown in Eq. (4).

$$
[m][\ddot{q}]+[C][\dot{q}]+[K][q]=[Q]
$$

where, $[Q]$ is column vector for all non-conservative forces, and $[q]$ is the column vector for all DOF which arranged as presented in Eq. (5). In addition, as a result, of straightforward choice for a set of minimum coordinates and the resultant vibration interferences for different masses, $[\mathrm{m}]$ is a square non-diagonal matrix for all components mass properties in the same order of the DOF.

$$
[q]=\left[\begin{array}{ccccccc}
y_{t F R} & y_{t F L} & y_{t M R} & y_{t M L} & y_{t B R} & y_{t B L} & \ldots \\
y_{T g} & \theta_{T g} & \varphi_{T g} & y_{d v} & \theta_{d v} & \varphi_{d v} & \ldots \\
y_{f p} & \theta_{f p} & \varphi_{f p} & y_{r p} & \theta_{r p} & \varphi_{r p} & \ldots \\
y_{f e R} & y_{f e L} & y_{f e B} & y_{f} & y_{o} & &
\end{array}\right]^{T}
$$

Finally, $[K]$ is stiffness skew symmetric matrix can be derived by calculating Eq. (6).

$$
K_{i j}=\frac{\partial^{2} V}{\partial q_{i} \partial q_{j}}
$$

Searching for the minimum resonance natural frequency for such simulated model, the eigenvalue problem presented in Eq. (7), in which, [ $\omega]$ is the resonance frequencies column vector, has to be solved.

$$
\operatorname{det}\left([K]-[\omega]^{2}[m]\right)=[0]
$$

As a result of, obvious equations complexity and the large number of variables, "Matlab" program will be implemented to construct both masses and stiffness matrices in addition to, calculate the minimum resonance frequency numerical value. The numerical parameters for such model are presented in article appendix

\section{SIMULATION RESULTS AND SENSITIVITY ANALYSIS}

In order to validate the constructed simulation results accuracy, the experimental modal test analysis was applied to calculate the minimum natural frequency for such model. Actually, such modal test was applied in order to calculate the longitudinal natural vibration where it's the dominant flexible vibration affects the firing accuracy. Of course, the main part affects the longitudinal vibration in this multibody model is the $T g$ part which represents both truck main body and cannon system. The simulation resultant minimum natural frequency based on the numerical values presented in the appendix is $10.86 \mathrm{~Hz}$. Naturally; this natural frequency is related to the rigid manner vibration behavior. On the other hand, the applied experimental test demonstrates that the minimum resonance longitudinal bending frequency is 4.45 Hz. Certainly, this result is not completely matched with the simulation result because it is measured in proportion to the real flexible model, but fortunately, it gives the signs for the approaching of this result from the real world, especially if the impact of the main parts flexibility nature of the weapon system were added which will converge the resultant results to the truth.

Sensitivity analysis is the famous optimization technique that has spread applications in the artillery designs. It helps the designer to distinguish the effect of variables variations on the system stability. Also, it denotes the critical variables for which additional information may be obtained searching for the optimal system layout. Three different design variable variations are selected to examine separately their effects on the Minimum Longitudinal Resonance Frequency (MLRF); stiffness values that simulate both suspension system and tires, the longitudinal position for both front and back ammunition depots, and finally the masses variation for both driver room and back ammunition depots. These sets were basically classified based on the designer experience for such weapon system family. Table III represents all design variables variations effect on the MLRF. For further clarification, Fig. 4 depicts all presented data in this table to determine the dominant critical value that effect MLRF. It's clear by studying Fig. (4) that, all design variables critically affect the MLRF especially in the maximum level of variation. For example in the case of stiffness value for the 
suspension system $K_{S}$, the simulation value must be greater than $150(\mathrm{k} . \mathrm{N} / \mathrm{m})$ to prevent any abrupt decrease in the MLRF. The critical range for the other selected design variables can be determined through such figure.

TABLE III: EFFECT OF DESIGN VARIABLE VARIATION ON THE MLRF

\begin{tabular}{|c|c|c|c|c|c|}
\hline Parameter & Value & MLRF & Parameter & Value & MLRF \\
\hline \multirow{8}{*}{ 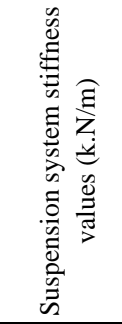 } & 5 & 3.2 & \multirow{8}{*}{ 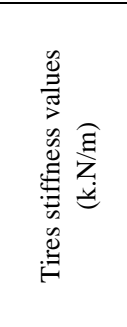 } & 5 & 4.1 \\
\hline & 50 & 7.9 & & 50 & 5.6 \\
\hline & 100 & 9.11 & & 100 & 7 \\
\hline & 200 & 9.52 & & 200 & 8.84 \\
\hline & 240 & 9.94 & & 210 & 9.93 \\
\hline & 300 & 9.96 & & 300 & 10.13 \\
\hline & 400 & 9.53 & & 400 & 12.3 \\
\hline & 500 & 9.97 & & 500 & 13.21 \\
\hline \multirow{7}{*}{$\zeta_{\mathrm{fp}}(\mathrm{cm})$} & 50 & 5.51 & \multirow{7}{*}{$\zeta_{\text {rp }}(\mathrm{cm})$} & 10 & 5.3 \\
\hline & 100 & 7.3 & & 50 & 8.84 \\
\hline & 150 & 9.1 & & 100 & 7.21 \\
\hline & 226 & 9.94 & & 151.5 & 9.94 \\
\hline & 300 & 10.1 & & 200 & 10.1 \\
\hline & 350 & 9.45 & & 250 & 9.44 \\
\hline & 400 & 8.18 & & 300 & 8.21 \\
\hline \multirow{7}{*}{$\mathrm{m}_{\mathrm{dv}}(\mathrm{lb})$} & 226.8 & 13.1 & \multirow{7}{*}{$\mathrm{m}_{\mathrm{rp}}(\mathrm{lb})$} & 226.8 & 12.1 \\
\hline & 362.9 & 11.35 & & 362.9 & 10.6 \\
\hline & 453.6 & 10.05 & & 453.6 & 10.01 \\
\hline & 593.3 & 9.94 & & 654.5 & 9.94 \\
\hline & 635 & 9.4 & & 657.7 & 9.1 \\
\hline & 657.7 & 7.65 & & 680.4 & 8.75 \\
\hline & 680.3 & 5.21 & & 703.1 & 7.91 \\
\hline
\end{tabular}

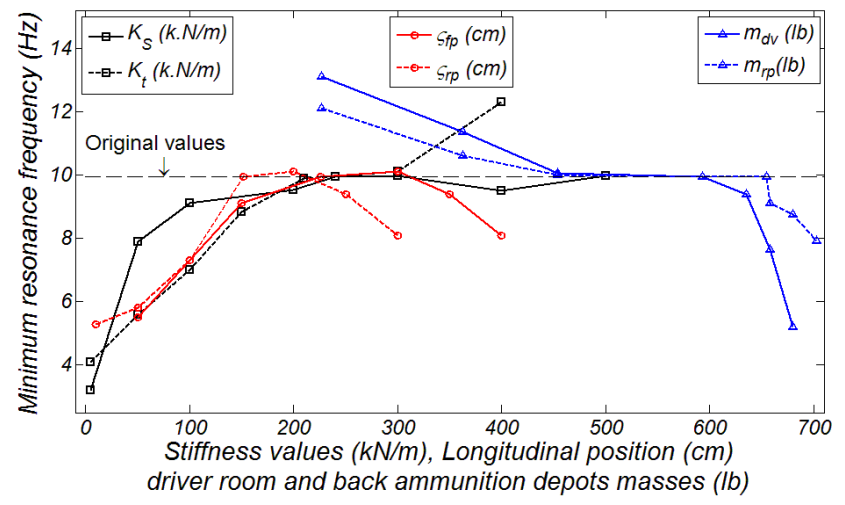

Fig. 4. Effect of design variable variation on the MLRF.

\section{CONCLUSION}

A technique of incorporating the Lagrangian approach to construct the multibody model in spatial coordinates to study the free vibration behavior for the truck mounted howitzer including the main components parameters has been developed. The equation of motion for such multibody model with 23 DOF has been derived directly. In order to calculate the natural frequency and mode shapes, the free vibration characteristic equation has been constructed. A sensitivity analysis for the constructed truck mounted howitzer model has been applied for different design variable sets, to investigate the best design parameters towards optimal weapon system design. Finally, this study has constructed the theoretic basis for the truck mounted howitzer system design, dynamic analysis, and layout finalization.

\section{APPENDIX: TECHNICAL PARAMETERS}

\begin{tabular}{|c|c|c|c|}
\hline \multicolumn{4}{|c|}{ Masses $(\mathrm{kg})$ and moments of inertia $\left(\mathrm{kg} \cdot \mathrm{m}^{2}\right)$ are } \\
\hline $\mathrm{m}_{\mathrm{Tg}}$ & 4023 & $\mathrm{I}_{\mathrm{Zrp}}$ & $5.75 \times 10^{3}$ \\
\hline $\mathrm{m}_{\mathrm{dv}}$ & 1308 & $I_{x d v}$ & $1.28 \times 10^{3}$ \\
\hline $\mathrm{m}_{\mathrm{fp}}$ & 3048 & $\mathrm{I}_{\mathrm{zdv}}$ & $1.33 \times 10^{3}$ \\
\hline $\mathrm{m}_{\mathrm{rp}}$ & 1443 & $\mathrm{I}_{\mathrm{xfp}}$ & $1.78 \times 10^{3}$ \\
\hline $\mathrm{m}_{\mathrm{feFR}}=\mathrm{m}_{\mathrm{feFL}}$ & 87 & $\mathrm{I}_{\mathrm{zfp}}$ & $2.31 \times 10^{2}$ \\
\hline $\mathrm{m}_{\mathrm{feB}}$ & 100 & $\mathrm{I}_{\mathrm{xrp}}$ & $1.13 \times 10^{3}$ \\
\hline $\mathrm{m}_{\mathrm{f}}=\mathrm{m}_{\mathrm{o}}$ & 120 & $\mathrm{I}_{\mathrm{zrp}}$ & $1.928 \times 10^{2}$ \\
\hline $\mathrm{m}_{\mathrm{t}}$ & 95.8 & $\mathrm{I}_{\mathrm{Xf}}$ & 1527 \\
\hline $\mathrm{I}_{\mathrm{XTg}}$ & $2.23 \times 10^{3}$ & $\mathrm{I}_{\mathrm{Zf}}$ & 6503 \\
\hline $\mathrm{I}_{\mathrm{ZTg}}$ & $1.35 \times 10^{4}$ & $\mathrm{I}_{\mathrm{X}_{\mathrm{O}}}$ & 1609 \\
\hline $\mathrm{I}_{\mathrm{Xdv}}$ & $1.28 \times 10^{3}$ & $\mathrm{I}_{\mathrm{Zo}}$ & 4986 \\
\hline $\mathrm{I}_{\mathrm{Zdv}}$ & $3.197 \times 10^{4}$ & $\mathrm{I}_{\mathrm{XfeFR}}=\mathrm{I}_{\mathrm{XfeFL}}$ & $5.23 \times 10^{3}$ \\
\hline $\mathrm{I}_{\mathrm{Xfp}}$ & $1.88 \times 10^{3}$ & $\mathrm{I}_{\mathrm{ZferR}}=\mathrm{I}_{\mathrm{ZfeFL}}$ & $3.75 \times 10^{3}$ \\
\hline $\mathrm{I}_{\mathrm{Zfp}}$ & $2.59 \times 10^{4}$ & $\mathrm{I}_{\mathrm{XfeB}}$ & $2.08 \times 10^{3}$ \\
\hline $\mathrm{I}_{\mathrm{Xrp}}$ & $1.45 \times 10^{3}$ & $\mathrm{I}_{\mathrm{ZfeB}}$ & $1.36 \times 10^{3}$ \\
\hline \multicolumn{4}{|c|}{ Stiffness values are(N/m) } \\
\hline $\mathrm{K}_{\mathrm{t}}$ & $2.4 \times 10^{5}$ & $\mathrm{~K}_{\mathrm{fp}}=\mathrm{K}_{\mathrm{rp}}$ & $55 \times 10^{3}$ \\
\hline $\mathrm{K}_{\mathrm{SB}}$ & $2.1 \times 10^{5}$ & $\mathrm{~K}_{\mathrm{feFR}}=\mathrm{K}_{\mathrm{feFL}}$ & $2.5 \times 10^{3}$ \\
\hline $\begin{array}{l}\mathrm{n}_{\mathrm{SB}} \\
\mathrm{K}_{\mathrm{SM}}\end{array}$ & $2.1 \times 10^{5}$ & $\begin{array}{l}\mathbf{\Lambda}_{\mathrm{feFR}}-\mathbf{\Lambda}_{\mathrm{feFL}} \\
\mathrm{K}_{\mathrm{feFB}}\end{array}$ & $2.5 \times 10^{3}$ \\
\hline $\mathrm{K}_{\mathrm{SF}}$ & $0.5 \times 10^{5}$ & $\mathrm{~K}_{\mathrm{f}}$ & $3.1 \times 10^{2}$ \\
\hline $\mathrm{K}_{\mathrm{dv}}$ & $44 \times 10^{5}$ & $\begin{array}{l}\mathrm{N}_{\mathrm{f}} \\
\mathrm{K}_{\mathrm{o}}\end{array}$ & $3.1 \times 10^{2}$ \\
\hline \multicolumn{4}{|c|}{ Geometry constants are (m) } \\
\hline$\zeta_{\mathrm{tFR}}=\zeta_{\mathrm{tFL}}$ & 4.536 & $\psi_{\mathrm{tFR}}=\psi_{\mathrm{tMR}}=\psi_{\mathrm{tBl}}$ & 0.941 \\
\hline$\zeta_{\mathrm{tMR}}=\zeta_{\mathrm{tML}}$ & 1.473 & \multirow{2}{*}{\multicolumn{2}{|c|}{$\psi_{\mathrm{tFL}}=\psi_{\mathrm{tML}}=\psi_{\mathrm{tBL}}$}} \\
\hline$\zeta_{\mathrm{tBR}}=\zeta_{\mathrm{tBL}}$ & 0.188 & & \\
\hline$\zeta_{\mathrm{dvBR}}=\zeta_{\mathrm{dvBL}}$ & 3.9595 & $\psi_{\mathrm{dvFL}}=\psi_{\mathrm{dvBL}}$ & 0.45 \\
\hline$\zeta_{\mathrm{dvFR}}=\zeta_{\mathrm{dvFL}}$ & 5.117 & $\psi_{\mathrm{dvFR}}=\psi_{\mathrm{dvBR}}$ & 0.47 \\
\hline $\mathrm{a}_{\mathrm{dvBR}}=\mathrm{a}_{\mathrm{dvBL}}$ & 1.24 & $b_{\mathrm{dvFL}}=b_{\mathrm{dvBL}}$ & 0.454 \\
\hline$a_{d v F R}=a_{d v F L}$ & 2.78 & $b_{\mathrm{dvFR}}=b_{\mathrm{dvBR}}$ & 0.462 \\
\hline$\zeta_{\mathrm{fpBR}}=\zeta_{\mathrm{fpBL}}$ & 2.26 & $\psi_{\mathrm{fpFL}}=\psi_{\mathrm{fpBL}}$ & 0.427 \\
\hline$\zeta_{\mathrm{fpFR}}=\zeta_{\mathrm{fpFL}}$ & 3.12 & $\psi_{\mathrm{fpFR}}=\psi_{\mathrm{fpBR}}$ & 0.406 \\
\hline $\mathrm{a}_{\mathrm{fpBR}}=\mathrm{a}_{\mathrm{fpBL}}$ & 0.45 & $\mathrm{~b}_{\mathrm{fpFL}}=\mathrm{b}_{\mathrm{fpBL}}$ & 0.382 \\
\hline $\mathrm{a}_{\mathrm{fpFR}}=\mathrm{a}_{\mathrm{fpFL}}$ & 0.42 & $\mathrm{~b}_{\mathrm{fpFR}}=\mathrm{b}_{\mathrm{fpBR}}$ & 0.45 \\
\hline$\zeta_{\mathrm{rpBR}}=\zeta_{\mathrm{rpBL}}$ & 1.515 & $\psi_{\mathrm{rpFL}}=\psi_{\mathrm{rpBL}}$ & 0.409 \\
\hline$\zeta_{\mathrm{rpFR}}=\zeta_{\mathrm{rpFL}}$ & 1.23 & $\psi_{\mathrm{rpFR}}=\psi_{\mathrm{rpBR}}$ & 0.431 \\
\hline $\mathrm{a}_{\mathrm{rpBR}}=\mathrm{a}_{\mathrm{rpBL}}$ & 0.392 & $\mathrm{~b}_{\mathrm{rpFL}}=\mathrm{b}_{\mathrm{rpBL}}$ & 0.416 \\
\hline $\mathrm{a}_{\mathrm{rpFR}}=\mathrm{a}_{\mathrm{rpFL}}$ & 0.252 & $\mathrm{~b}_{\mathrm{rpFR}}=\mathrm{b}_{\mathrm{rpBR}}$ & 0.424 \\
\hline$\zeta_{\mathrm{feFR}}$ & 1.86 & $\psi_{\mathrm{feFR}}$ & 1.029 \\
\hline$\zeta_{\mathrm{feFL}}$ & 1.86 & $\psi_{\mathrm{feFL}}$ & 1.008 \\
\hline$\zeta_{\mathrm{feB}}$ & 0.815 & $\psi_{\mathrm{feB}}$ & 0.011 \\
\hline$\zeta_{\mathrm{f}}$ & 2.471 & $\psi_{\mathrm{f}}$ & 0.607 \\
\hline$\zeta_{0}$ & 1.167 & $\psi_{\mathrm{o}}$ & 0.183 \\
\hline
\end{tabular}

\section{REFERENCES}

[1] A. Fasano and S. Marmi, Analytical Mechanics, 1st ed. Oxford University Press, 2006, ch. 4, pp.140-182.

[2] R. F. Grant and L. G. Cassiday, Analytical Mechanics, Saunders college, 1999, ch. 9, pp. 361-409.

[3] S. Bernd, Computational Flexible Multibody Dynamics: A Differential-Algebraic Approach, Springer Science and Business Media, Berlin, 2013, ch. 2, 3, pp.13-156

[4] F. A. Alexandr, V. Kabanau, and V. Vantsevich, Driveline Systems of Ground Vehicles: Theory and Design, CRC Press, 2010, pp. 1-114.

[5] Category. [Online]. Available: https://en.wikipedia.org/wiki/Category:Military_trucks

[6] N. Jazar, Vehicle Dynamics. Theory and Applications, Riverdale, Springer Science, New York, 2008, ch. 13, pp. 827-878.

[7] S. Zhong, D. Zhao, Y. Sun, and Q. Wei, "Modeling and modal analysis of truck chassis based on FEM," Journal of Machinery Design and Manufacture, vol. 6, Jan. 2008.

[8] S. Bernd, F. Grupp, C. Führer, and P. Rentrop, "A nonlinear truck model and its treatment as a multibody system," Journal of Computational and Applied Mathematics, vol. 1, pp. 523-532, May 1994.

[9] I. M. Ibrahim, D. A. Crolla, and D. C. Barton, "Effect of frame flexibility on the ride vibration of trucks," Journal of Computers \& structures, vol. 58, no. 4, pp. 709-713, Feb. 1996.

[10] A. R. Ragaee, G. L. Yang, and J. L. Ge, "Modal analysis for a complex military trucks structure," Journal of Vibroengineering, vol.17, no. 6 , pp. 3147-3159, Sep. 2015. 
[11] W. Y. Cai, Y. S. Chen, and G. L. Yang, "Impacts of structural parameters on muzzle disturbance for a vehicle mounted howitzer," Journal of Nanjing University of Science and Technology, vol. 29, no. 6, pp. 658-662, Jun. 2005.

[12] W. Y. Cai, "Large caliber artillery flexible multi-vehicle dynamics and overall optimization of the body," Ph.D. dissertation, Dept. Mech. Eng., NUST Univ., Nanjing, 2011.

[13] H. L. Yu and X. T. Rui. (January 2014). Study on launch dynamics of self-propelled artillery based on transfer matrix method of multibody system. Advances in Mechanical Engineering, 6. [Online]. Available: http://ade.sagepub.com/content/6/308049.full\#corresp-1

[14] A. Jorge and A. Kecskeméthy, Kinematics and Dynamics of Multi-Body Systems, vol. 360, pp. 11-75.

Ragaee Rateb was admitted to study a PhD degree in School of Mechanical Engineering from Nanjing University of Science and Technology, Nanjing, China, in 2014. Now he is still doing research in NUST. His current research interests include numerical simulation, multi-body dynamics, hydraulic control.

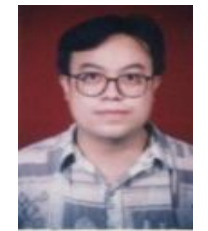

Guolai Yang received his $\mathrm{PhD}$ degree in School of Mechanical Engineering from Nanjing University of Science and Technology, Nanjing, China, in 1999 Now he works as a professor at NUST. His current research interests include multi-body system dynamics, nonlinear vibration and control.

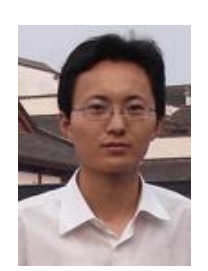

Jianle Ge received his $\mathrm{PhD}$ degree in School of Mechanical Engineering from Nanjing University of Science and Technology, Nanjing, China, in 2007. Now he works as an associated professor in NUST. His current research numerical simulation, dynamic analysis, and applied mechanics. 